\title{
Posterior Hyaloidotomy by Nd:YAG Laser Application in a Patient with Postpartum Depression Caused by Valsalva Retinopathy
}

\author{
Mutlu Cihan Dağlıoğlu ${ }^{a} \quad$ Mesut Coşkun ${ }^{a} \quad$ Nilüfer İlhan ${ }^{a}$ \\ Esra Ayhan Tuzcu ${ }^{a}$ Mustafa Arı ${ }^{b}$ Emre Ayıntap ${ }^{c}$ Özgür İlhan ${ }^{a}$ \\ Departments of ${ }^{a}$ Ophthalmology and ${ }^{b}$ Psychiatry, Mustafa Kemal University, Faculty of \\ Medicine, Antakya, and 'Department of Ophthalmology, Bezmi Alem University, \\ Istanbul, Turkey
}

\section{Key Words}

Nd:YAG laser $\cdot$ Postpartum depression · Pregnancy $\cdot$ Valsalva retinopathy

\begin{abstract}
Purpose: The aim of this report is to present a case of premacular hemorrhage in a 37-week pregnant woman with concurrent presence of postpartum depression secondary to her ocular disease. Methods: A 27-year-old woman in her 37th week of pregnancy presented with Valsalva retinopathy, which occurred in her left eye secondary to severe coughing. Her visual acuity was reduced to perception of hand motions, and she was agitated because of the loss of vision. The patient, who gave birth by cesarean section, was admitted for control examination 2 weeks after birth. No change was observed in premacular hemorrhage in control examination. However, the patient's relatives stated that the patient was very agitated and disinterested in her baby. For this reason, the patient was referred to the psychiatry clinic, and she was diagnosed with postpartum depression. The anatomical location of the premacular hemorrhage was determined by spectral domain optical coherence tomography. Results: Neodymium yttrium aluminum garnet ( $\mathrm{Nd}: \mathrm{YAG})$ laser hyaloidotomy was performed to her left eye on the same day. Rapid release of premacular hemorrhage into the vitreous cavity was observed immediately after Nd:YAG laser application. Two days after the application, the patient's visual acuity had improved to 20/20. The clinical improvement positively impacted on the patient's mood. Conclusion: In pregnant patients suffering from Valsalva retinopathy during the postpartum period, posterior hyaloidotomy by Nd:YAG laser
\end{abstract}


Dağlıoğlu et al.: Posterior Hyaloidotomy by Nd:YAG Laser Application in a Patient with Postpartum Depression Caused by Valsalva Retinopathy

can be implemented in terms of the patients' own and their babies' physical and mental health.

\section{Introduction}

Valsalva retinopathy is a unilateral or bilateral condition characterized with sudden loss of vision due to premacular hemorrhage. Sudden rise in intra-abdominal or intrathoracic pressure may cause a rapid increase in venous pressure with spontaneous rupture of superficial retinal capillaries. The patient often gives a history of recent heavy lifting, straining on the toilet, vomiting, coughing or labor [1].

Pregnancy is considered to be a common risk factor for Valsalva retinopathy. Risk factors for hemorrhage and Valsalva retinopathy are increased in pregnancy due to the hormonal, metabolic, hematological and immunological alterations. Elevation of intraabdominal pressure during pregnancy because of an enlarging uterus leads to elevation in intravenous pressure, which also decreases venous return [2]. In Valsalva retinopathy, hemorrhage may be subhyaloid, under the internal limiting membrane or both [3]. Rarely, it can be seen between the internal limiting membrane and nerve fiber layer, which is selflimiting because of the anatomical structure. Neodymium yttrium aluminum garnet (Nd:YAG) laser is commonly used for the treatment of subhyaloid hemorrhages [4]. Additionally, spontaneous resolution can be seen in certain cases [5]. However, the presence of additional pathologies caused by vision loss might require early intervention.

Here, we present a case of a postpartum depression patient suffering from premacular hemorrhage treated with application of Nd:YAG laser posterior hyaloidotomy. In order to avoid retinal tissue damage, anatomical localization of hemorrhage was detected using spectral domain optical coherence tomography (SD-OCT) which was later used for follow-up following laser application as well.

\section{Case History}

A 27-year-old woman at 37 weeks of pregnancy presented to our department with sudden vision loss in her left eye for 1 week. She had no history of medication use or trauma. Hematological, immunological and hormonal tests were within normal limits. The patient stated that she had severe coughing for a few days before loss of vision. The right eye was completely normal. Visual acuity was reduced to perception of hand motions in the left eye. Globe movements and intraocular pressure were normal in both eyes. Fundus examination of the right eye was normal. However, fundus examination of the left eye showed premacular hemorrhage.

Color fundus photograph showed a massive premacular hemorrhage that was fivefold the macular diameter. Nearly at the end of the pregnancy period and for the possibility of spontaneous resolution the patient was followed without intervention. One week later the patient gave birth by cesarean section. There were no changes in the complaint and symptoms of the patient in the control examination 2 weeks after birth. At the control examination, hemosiderin deposition was observed between the detached hyaloid membrane and retina. Retinal layers were detected to be intact and the hemorrhage was found in the subhyaloid region using SD-OCT (RTVUE-100 Fourier Spectral Domain Optical Coherence Tomography, Optovue, Inc., Fremont, Calif., USA) (fig. 1). Interviewing the relatives of the patient revealed that she was disinterested in her baby, agitated, continuous- 
Dağlıoğlu et al.: Posterior Hyaloidotomy by Nd:YAG Laser Application in a Patient with Postpartum Depression Caused by Valsalva Retinopathy

ly crying and constantly complaining about the vision loss. Furthermore, she suffered from insomnia and an extreme decrease in self-care and self-esteem. She was referred to the psychiatry department. The Edinburgh Postnatal Depression Scale (EPDS) was administered and she scored 32 points [6]. Since the cutoff point for the scale is $12 / 13$ she was diagnosed with postpartum depression, and the complaints were attributed to her depression most probably induced by vision loss secondary to premacular hemorrhage. Psychotropic medication was not given to the patient because she breastfed her baby.

The hyaloid membrane was perforated with two rounds of Nd:YAG laser with $2.4 \mathrm{~mJ}$ energy. Consequently, the hemorrhage was rapidly released into the vitreous cavity (fig. 2). Two days after the application, the patient's visual acuity improved to $20 / 20$. The psychiatric status of the patient dramatically improved and the EPDS score was reduced to 11 following operation. Although a full withdrawal of hemorrhage occurred, a minimal subhyaloid cavitation was still observable on SD-OCT (fig. 3). Furthermore, laser perforation points incurred during the procedure were not observed in the last SD-OCT.

\section{Discussion}

Valsalva retinopathy is a relatively rare entity characterized by premacular retinal hemorrhage. Determining the exact location of the hemorrhage is very important in terms of the efficacy of the procedure. Therefore, SD-OCT that provides clear cross-sectional images is commonly used today. In addition, it is also an ideal method to observe changes of the hyaloid membrane and retinal layers following laser application.

Different approaches have been reported about Valsalva retinopathy occurring during pregnancy [2, 5, 7]. El-Defrawy et al. [5] preferred a conservative approach considering spontaneous resolution of retinal hemorrhage in two pregnant women with retinal hemorrhage. The first case showed 6/60 visual acuity due to retinal hemorrhage. Two weeks after delivery her vision had spontaneously improved to $6 / 6$ in the left eye with near-total resolution of the retinal and vitreous hemorrhage. However, the second case showed complete resolution of the subhyaloid hemorrhage 5 months after delivery [2]. Besides follow-up without intervention, there are reports presenting cases treated with Nd:YAG laser hyaloidotomy 2 weeks after birth [7].

A woman at 37 weeks of pregnancy presented to our department with premacular hemorrhage. She was extremely agitated because of sudden vision loss in her left eye. A constant request of immediate treatment was refused by our team. The patient was convinced that she would be treated with laser application after delivery. An Nd:YAG laser procedure was performed 2 weeks after delivery because of postpartum depression induced by vision loss. Two shots of laser were applied to the hemorrhagic region on a site away from the macula. Consequently, the hemorrhage was rapidly released into the vitreous cavity. Two days after the application, the patient's visual acuity improved to 20/20.

$\mathrm{Nd}: Y A G$ laser posterior hyaloidotomy is commonly applied in premacular hemorrhage treatment, which has lately become even safer with the help of SD-OCT. In the literature, there is not sufficient data regarding the safe use of YAG laser in pregnant women with Valsalva retinopathy. However, in cases suffering from massive hemorrhage Nd:YAG laser can be applied at an appropriate time after the delivery. In the presented case Nd:YAG laser hyaloidotomy was performed shortly after the delivery because of the postpartum depression leading to reduced self-care and disinterest in her baby.

In conclusion, pregnant patients suffering from Valsalva retinopathy are recommended to undergo Nd:YAG laser posterior hyaloidotomy as soon as possible in order to avoid 
Dağlıoğlu et al.: Posterior Hyaloidotomy by Nd:YAG Laser Application in a Patient with Postpartum Depression Caused by Valsalva Retinopathy

aggravated depression that might deteriorate the patients' own and their babies' physical and mental health.

\section{Disclosure Statement}

The authors have no financial interest in any of the products mentioned in this case report.

\section{References}

1 Chapman-Davies A, Lazarevic A: Valsalva maculopathy. Clin Exp Optom 2002;85:42-45.

1 Al-Mujaini AS, Montana CC: Valsalva retinopathy in pregnancy: a case report. J Med Case Rep 2008;2:101.

3 Gass JDM: Stereoscopic Atlas of Macular Disease: Diagnosis and Treatment, ed 4. St. Louis, Mosby, 1997, pp 752-754.

4 Sabella P, Bottoni F, Staurenghi G: Spectral-domain OCT evaluation of Nd:YAG laser treatment for Valsalva retinopathy. Graefes Arch Clin Exp Ophthalmol 2010;248:599-601.

5 El-Defrawy H, Sallam A, McKechnie CJ, Zambarakji H: Valsalva retinopathy in pregnancy. Should we treat? JRSM Short Rep 2011;2:45.

6 Cox JL, Holden JM, Sagovsky R: Detection of postnatal depression. Development of the Edinburgh Postnatal Depression Scale (EPDS). Br J Psychiatry 1987;150:782-786.

7 Ladjimi A, Zaouali S, Messaoud R, et al: Valsalva retinopathy induced by labour. Eur J Ophthalmol 2002;12:336-338.

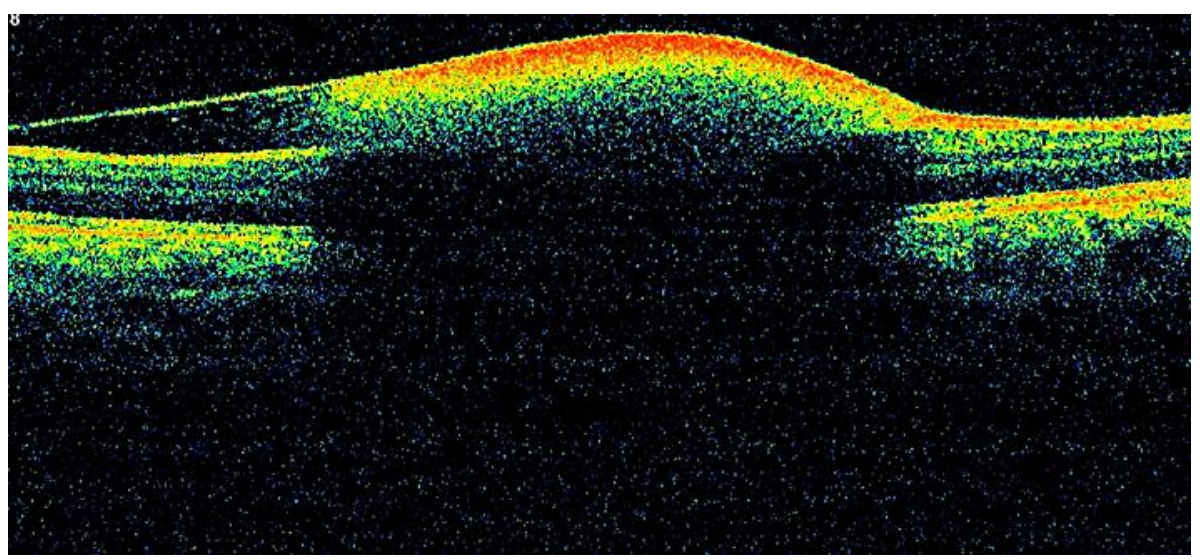

Fig. 1. Plasma under the hyaloid membrane in the upper section. Shadowing due to concentrated blood cells in the lower section. 
Case Reports in

Ophthalmology
Case Rep Ophthalmol 2013;4:64-68

DOI: $10.1159 / 000350773$

Dağlıŏlu et al.: Posterior Hyaloidotomy by Nd:YAG Laser Application in a Patient with

Postpartum Depression Caused by Valsalva Retinopathy

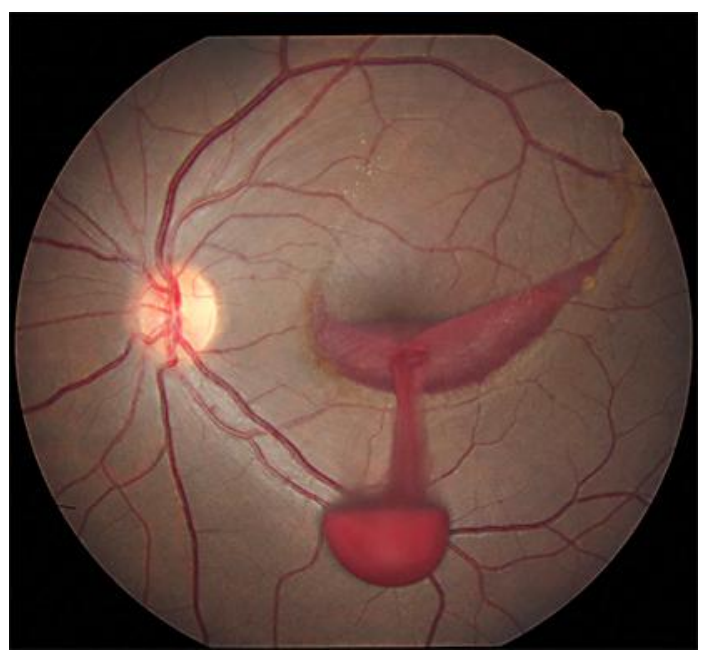

Fig. 2. Rapid release of blood to the vitreous cavity after Nd:YAG laser shots to the detached hyaloid membrane.

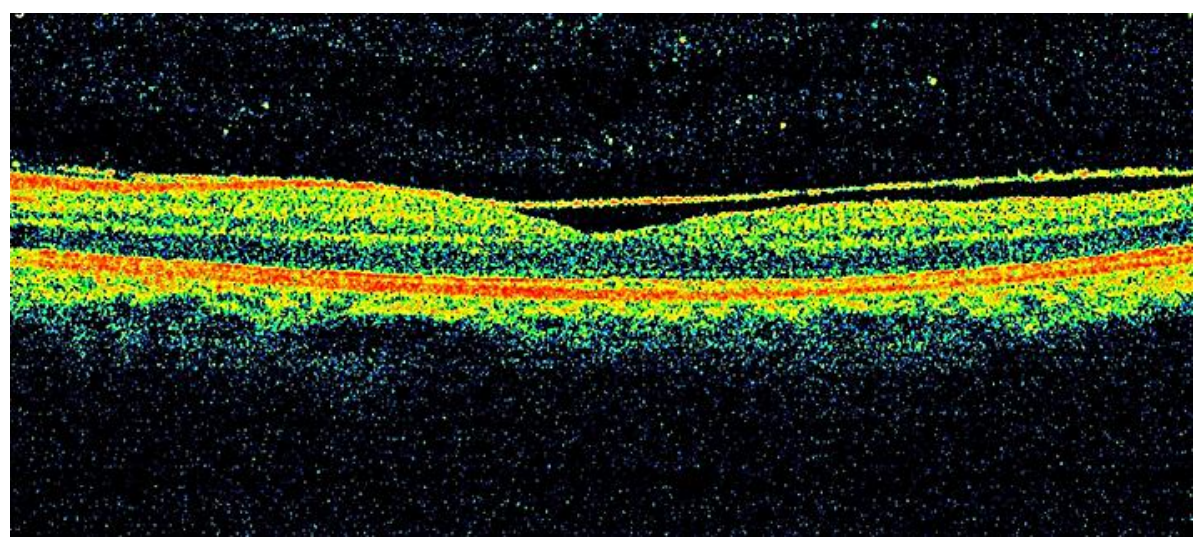

Fig. 3. Minimal cavitation between the internal limiting membrane and intact retina 1 month after Nd:YAG laser application. 